\title{
Checkerboard Julia Sets for Rational Maps
}

\author{
Paul Blanchard \\ Figen Çilingir* \\ Daniel Cuzzocreo \\ Robert L. Devaney \\ Daniel M. Look \\ Elizabeth D. Russell \\ Current version: June 16, 2011 \\ Original draft: February 8, 2010
}

\begin{abstract}
In this paper, we consider the family of rational maps

$$
F_{\lambda}(z)=z^{n}+\frac{\lambda}{z^{d}}
$$

where $n \geq 2, d \geq 1$, and $\lambda \in \mathbb{C}$. We consider the case where $\lambda$ lies in the main cardioid of one of the $n-1$ principal Mandelbrot sets in these families. We show that the Julia sets of these maps are always homeomorphic. However, two such maps $F_{\lambda}$ and $F_{\mu}$ are conjugate on these Julia sets only if the parameters at the centers of the given cardioids satisfy $\mu=\nu^{j(d+1)} \lambda$ or $\mu=\nu^{j(d+1)} \bar{\lambda}$ where $j \in \mathbb{Z}$ and $\nu$ is an $n-1^{\text {st }}$ root of unity. We define a dynamical invariant, which we call the minimal rotation number. It determines which of these maps are conjugate on their Julia sets, and we obtain an exact count of the number of distinct conjugacy classes of maps drawn from these main cardioids.
\end{abstract}

*The second author would like to thank the Department of Mathematics and Statistics at Boston University for its hospitality while this work was in progress. In addition, she would also like to thank TUBITAK for their support while this research was in progress. 
In recent years there have been many papers dealing with the family of rational maps given by

$$
F_{\lambda}(z)=z^{n}+\frac{\lambda}{z^{d}}
$$

where $n \geq 2, d \geq 1$, and $\lambda \in \mathbb{C}[9]$. For many parameter values, the Julia sets for these maps are Sierpiński curves, i.e., planar sets that are homeomorphic to the well-known Sierpiński carpet fractal. One distinguishing property of Sierpiński curve Julia sets is that the Fatou set consists of infinitely many open disks, each bounded by a simple closed curve, but no two of these bounding curves intersect.

There are many different ways in which these Sierpiński curves arise as Julia sets in these families. For example, the Julia set is a Sierpiński curve if $\lambda$ is a parameter for which

1. the critical orbits enter the immediate basin of attraction of $\infty$ after two or more iterations [5];

2. the parameter lies in the main cardioid of a "buried" baby Mandelbrot set [3]; or

3. the parameter lies on a buried point in a Cantor necklace in the parameter plane [7].

The parameter planes for these maps in the cases where $n=d=3$ and $n=d=4$ are shown in Figure 1. The red disks not centered at the origin are regions where the first case above occurs. These disks are called Sierpiński holes.

Many Mandelbrot sets are visible in Figure 1. The ones that touch the external red region are not "buried," so their main cardioids do not contain Sierpiński curve Julia sets. Only the ones that do not meet this boundary contain parameters from case 2 .

Finally, numerous Cantor necklaces, i.e., sets homeomorphic to the Cantor middle-thirds set with the removed open intervals replaced by open disks, appear in these figures. The buried points in the Cantor set portion of the necklace are the parameters for which case 3 occurs. 

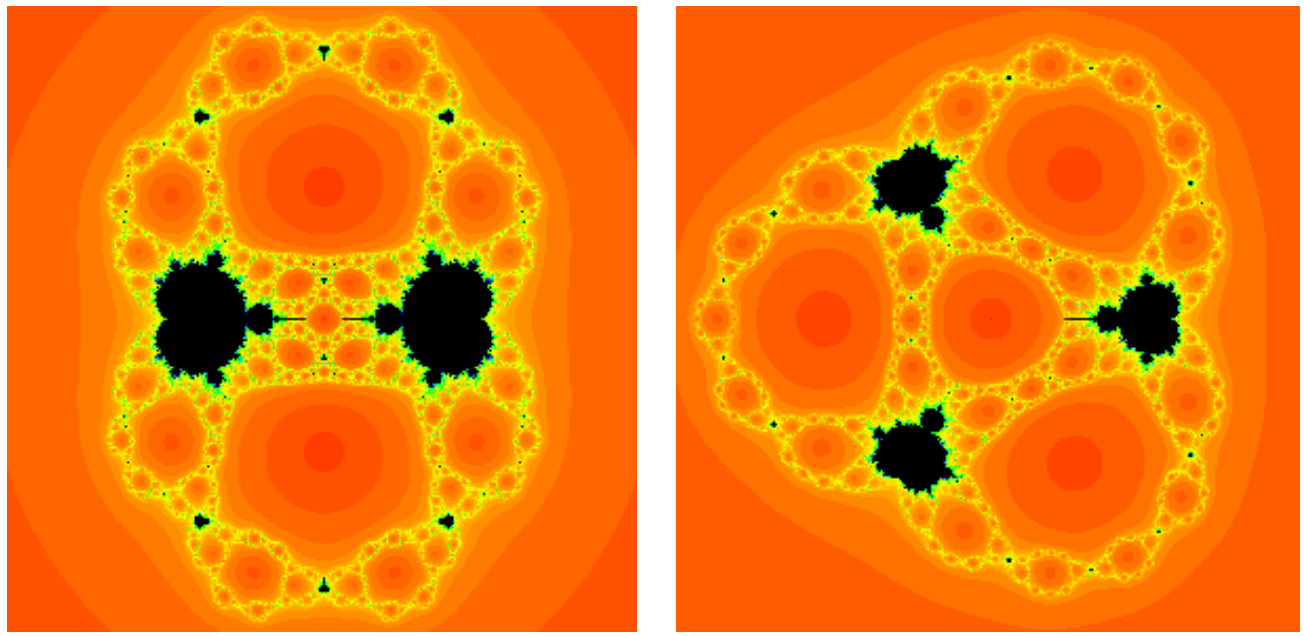

Figure 1: Two parameter planes: $n=d=3$ (left) and $n=d=4$ (right).

The dynamical behavior on Sierpiński curve Julia sets drawn from nonsymmetrically located Sierpiński holes is never the same [6]. That is, only symmetrically located Sierpiński holes contain parameters for which the corresponding maps have conjugate dynamics. While it is known that two such maps are not conjugate on their Julia sets, there is no known dynamical invariant that explains this lack of conjugacy.

In this paper, we describe the topology of and dynamics on a very different type of Julia set that arises in these families, the "checkerboard" Julia sets. The parameter spaces contain $n-1$ "principle Mandelbrot sets," the largest Mandelbrot sets in the parameter space [8]. We consider the Julia sets for the parameters that lie in the main cardioids of these Mandelbrot sets.

Parameters from these cardioids have two distinct types of Fatou components (see Figure 2). Since $\infty$ is a superattracting fixed point, the immediate basin of $\infty$ and its preimages lie in the Fatou set. These components are the escaping Fatou components. The Fatou set also contains a collection of components corresponding to other finite attracting periodic orbits and their preimages. These components are the non-escaping Fatou components. As we shall show, none of the boundaries of the escaping Fatou components 
intersect. Likewise, the boundaries of the non-escaping Fatou components do not intersect. However, each such boundary intersects infinitely many boundaries of the escaping Fatou components, and each boundary of an escaping Fatou component intersects infinitely many non-escaping boundaries. Hence, the topology of these Julia sets is very different from the topology of Sierpiński curve Julia sets. We use the word "checkerboard" to describe this pattern of Fatou components.

In Figure 2, we display the Julia set for the map $F_{0.18}(z)=z^{4}+0.18 / z^{3}$. The red regions are the preimages of the attracting basin of $\infty$, and the black regions are the preimages of the basins of the finite attracting cycles. The boundary of each red region touches infinitely many boundaries of the black regions, but it does not touch the boundary of any other red region. Similarly, the boundary of each black region touches infinitely many boundaries of the red regions, but it does not touch the boundary of any other black region.
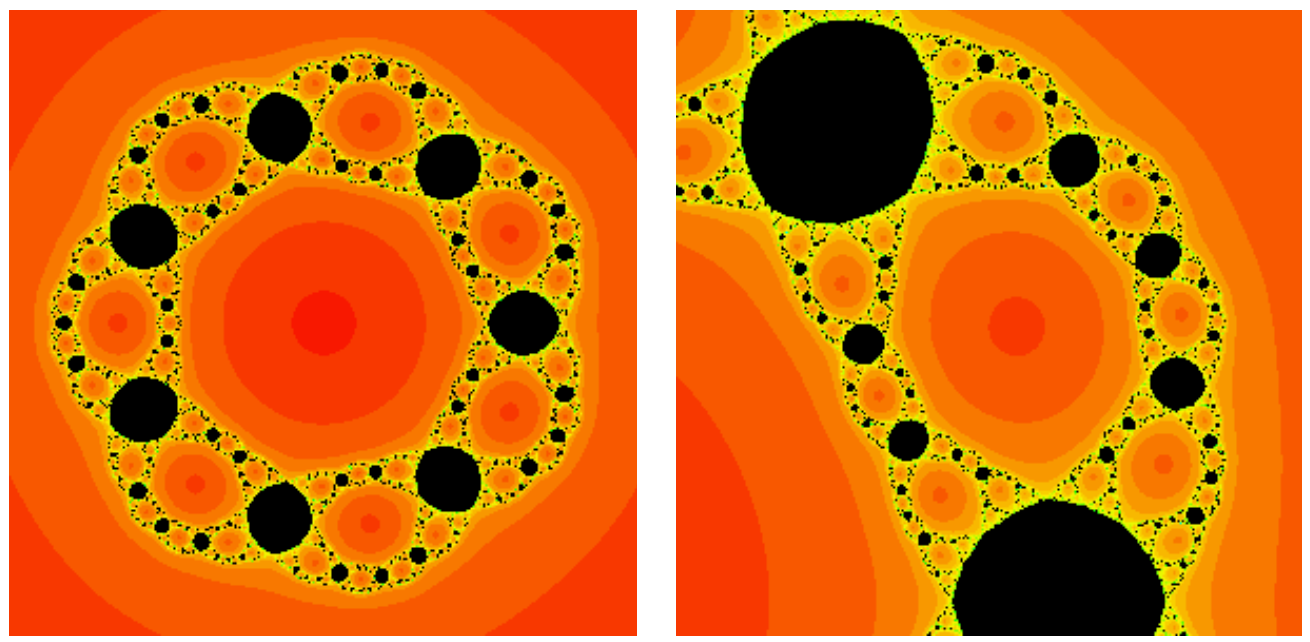

Figure 2: The image on the left is the checkerboard Julia set for $F_{0.18}(z)=$ $z^{4}+0.18 / z^{3}$. The image on the right is a magnification of one-seventh of the Julia set.

The external red region in the left-hand image of Figure 2 is the immediate basin of attraction of $\infty$. We denote this basin by $B_{\lambda}$. The central red region 
that contains the pole at the origin is mapped to $B_{\lambda}$. We let $T_{\lambda}$ denote this Fatou component. All other red regions are also eventually mapped to $B_{\lambda}$.

Note that there are $n+d(=7$ in this example) large black regions that touch $B_{\lambda}$ and $T_{\lambda}$ at unique points. These Fatou components are (eventually) periodic. We call them the connecting (Fatou) components since they are the only Fatou components that extend from $B_{\lambda}$ to $T_{\lambda}$. Each of these connecting components seems to be separated by another red region that touches exactly two boundaries of the adjacent connecting components. On one side of these red regions, we see $d-1$ (=2 in this example) smaller black components. On the other side, we see $n-1$ (=3 in this example) smaller black components. Each such black component connects to a pair of red regions. If we were to magnify this image, we would see that this pattern repeats itself at any scale.

In this paper, we make this construction precise. In particular, we give an algorithm for describing the topological structure of these Julia sets. This algorithm also describes the dynamics on these Julia sets via symbolic dynamics. We prove:

Theorem 1. Let $F_{\lambda}(z)=z^{n}+\lambda / z^{d}$ with $n \geq 2$ and $d \geq 1$. Any two Julia sets that correspond to parameters in the main cardioids of the principal Mandelbrot sets in the parameter plane for these maps are homeomorphic.

Theorem 1 says that checkerboard Julia sets are analogous to Sierpiǹski curve Julia sets [15] because all checkboard Julia sets are homeomorphic.

As in the Sierpiǹski case [6], only certain symmetrically located cardioids give rise to conjugacies on their respective Julia sets. However, unlike the Sierpiǹski case, we can define a dynamical invariant for checkboard Julia sets. We call it the minimal rotation number, and we prove that it is a conjugacy invariant for checkerboard Julia sets.

Theorem 2. Two maps drawn from different main cardioids of principal Mandelbrot sets are topologically conjugate on their Julia sets if and only if their minimal rotation numbers are equal. In particular, two such maps restricted to their Julia sets are topologically conjugate only if the parameters 
are symmetric either under the rotation $z \mapsto \nu^{j(d+1)} z$ or under the map $z \mapsto \nu^{j(d+1)} \bar{z}$, where $j \in \mathbb{Z}$ and $\nu^{n-1}=1$.

Theorem 2 leads to an exact count of the number of main cardioids that have non-conjugate dynamics.

Theorem 3. Let $g$ be the greatest common divisor of $n-1$ and $n+d$. If $g$ is even, then there are exactly $1+g / 2$ distinct conjugacy classes among the maps drawn from the main cardioids of the principal Mandelbrot sets. If $g$ is odd, then the number of conjugacy classes is $(g+1) / 2$.

\section{Preliminaries}

Consider the family of maps

$$
F_{\lambda}(z)=z^{n}+\frac{\lambda}{z^{d}}
$$

where $n \geq 2, d \geq 1$, and $\lambda \in \mathbb{C}$. The point at infinity is superattracting of order $n$. As above, we denote the immediate basin of $\infty$ by $B_{\lambda}$. Also, 0 is a pole of order $d$, so there is a neighborhood of 0 that is mapped into $B_{\lambda}$. If this neighborhood is disjoint from $B_{\lambda}$, we use the term "trap door" for the preimage of $B_{\lambda}$ that contains 0 . We denote the trap door by $T_{\lambda}$.

The map $F_{\lambda}(z)$ has $n+d$ "free" critical points. They satisfy the equation

$$
z^{n+d}=\frac{d \lambda}{n} .
$$

Hence, they are equally spaced on the circle of radius

$$
\sqrt[n+d]{\frac{d|\lambda|}{n}}
$$

centered at the origin. There are also $n+d$ prepoles. They satisfy the equation $z^{n+d}=-\lambda$.

The family $F_{\lambda}$ has symmetries in both the dynamical plane and the parameter plane. 
Symmetry Lemma 1. The map $F_{\lambda}$ is conjugate to $F_{\bar{\lambda}}$ by the conjugacy $z \mapsto \bar{z}$.

This first symmetry implies that the parameter plane is symmetric under complex conjugation.

Symmetry Lemma 2. If $\omega$ is a $(n+d)$ th root of unity, then

$$
F_{\lambda}(\omega z)=\omega^{n} F_{\lambda}(z) .
$$

This second symmetry implies that the Julia set of $F_{\lambda}$ is symmetric under the map $z \mapsto \omega z$. Similarly, $B_{\lambda}$ and $T_{\lambda}$ possess this $(n+d)$-fold symmetry.

Moreover, since the free critical points are arranged symmetrically with respect to $z \mapsto \omega z$, all of the free critical orbits behave symmetrically with respect to this rotation. However, it is not necessarily true that all of these critical orbits behave in the same manner. For example, consider the map $F_{0.18}(z)=z^{4}+0.18 / z^{3}$ (see Figure 2). The orbit of the free critical point on the positive real axis is asymptotic to a fixed point, but the other six free critical orbits are asymptotic to a pair of period-three orbits. This symmetry also implies that the basins of these attracting orbits are arranged symmetrically as well.

The most important consequence of Symmetry Lemma 2 is the fact that the orbits of all of the free critical points can be determined from the orbit of any one of them (see Figure 3). So the one-dimensional $\lambda$-plane is a natural parameter plane for these maps.

Symmetry Lemma 3. Suppose that $\eta$ is an $(n+d)(n-1)$ st root of unity. Let $\nu=\eta^{n+d}$ and $\omega=\eta^{n-1}$. Then

$$
F_{\nu \lambda}^{k}(\eta z)=\eta^{n^{k}} F_{\lambda}^{k}(z)
$$

for $k=1,2,3, \ldots$

Note that $\nu$ is an $(n-1)$ st root of unity and $\omega$ is an $(n+d)$ th root of unity. Symmetry Lemma 3 is proved by induction on $k$. 


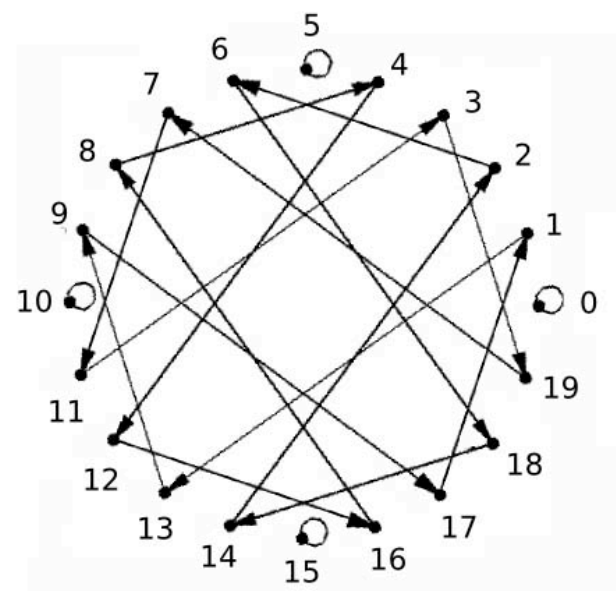

Figure 3: The orbit diagram for the critical points of $F_{\lambda_{0}}(z)=z^{13}+\lambda_{0} / z^{7}$ where $\lambda_{0}$ is the center of the principal Mandelbrot set $\mathcal{M}_{0}$ that intersects the positive real axis. The critical point on the positive real axis is a fixed point, and it is labeled with the number 0 . The orbits of the remaining critical points are determined from the orbit of the fixed point using Symmetry Lemma 2.

We can determine the orbit diagram of $F_{\nu \lambda}$ from the orbit diagram of $\lambda$. In particular, if $c_{\lambda}$ is a critical point for $F_{\lambda}$, then $\eta c_{\lambda}$ is a critical point for $F_{\nu \lambda}$. We denote this critical point by $c_{\nu \lambda}$. From Symmetry Lemma 3, we have

$$
F_{\nu \lambda}^{k}\left(c_{\nu \lambda}\right)=F_{\nu \lambda}^{k}\left(\eta c_{\lambda}\right)=\eta^{n^{k}} F_{\lambda}^{k}\left(c_{\lambda}\right)
$$

Therefore, the orbits of the critical points of $F_{\lambda}$ and $F_{\nu \lambda}$ behave symmetrically with respect to rotation by some power of $\eta$ (see Figure 4). Consequently, the parameter plane is symmetric under the rotation $\lambda \mapsto \nu \lambda$.

As we shall see, the dynamics of $F_{\lambda}$ and $F_{\nu \lambda}$ are not necessarily conjugate. For example, if $\lambda$ lies in the main cardioid of the right-hand principal Mandelbrot set in the $n=d=3$ case, the map $F_{\lambda}$ has a pair of attracting fixed points (see Figure 1). In contrast, if $\lambda$ is an element of the the main cardioid of the left-hand principal Mandelbrot set, the map $F_{\lambda}$ has an attracting cycle of period two. 


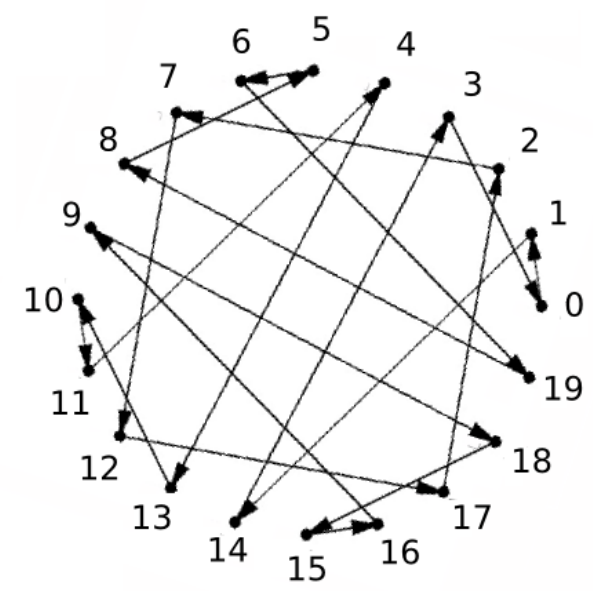

Figure 4: The orbit diagram for the critical points of $F_{\lambda_{1}}(z)=z^{13}+\lambda_{1} / z^{7}$ where $\lambda_{1}=\nu \lambda_{0}$ is the center of the principal Mandelbrot set $\mathcal{M}_{1}$, i.e., the image of $\mathcal{M}_{0}$ under the rotation $z \mapsto \nu z$.

The map $F_{\nu \lambda}$ is conjugate to $\omega F_{\lambda}$ by the rotation $z \mapsto \eta z$ because

$$
F_{\nu \lambda}(\eta z)=(\eta z)^{n}+\frac{\nu \lambda}{(\eta z)^{d}}=\eta^{n} z^{n}+\frac{\nu}{\eta^{d}} \frac{\lambda}{z^{d}}=\eta^{n}\left(z^{n}+\frac{\lambda}{z^{d}}\right)=\eta \omega\left(z^{n}+\frac{\lambda}{z^{d}}\right) .
$$

More generally, the map $F_{\nu^{j} \lambda}$ is conjugate to the map $\omega^{j} F_{\lambda}$ via the rotation $z \mapsto \eta^{j} z$.

As in the case of the quadratic polynomials $z^{2}+c$, the orbits of the free critical points can tend to $\infty$. However, unlike the quadratic case, there are three distinct ways that these critical orbits can escape, and each way leads to a different topological type of Julia set.

Theorem (The Escape Trichotomy [5].) Let $v_{\lambda}=F_{\lambda}\left(c_{\lambda}\right)$ be a critical value.

1. If $v_{\lambda}$ lies in $B_{\lambda}$, then $J\left(F_{\lambda}\right)$ is a Cantor set.

2. If $T_{\lambda} \neq B_{\lambda}$ and $v_{\lambda}$ lies in $T_{\lambda}$, then $J\left(F_{\lambda}\right)$ is a Cantor set of disjoint, simple, closed curves that surround the origin. 
3. In all other cases, $J\left(F_{\lambda}\right)$ is a connected set. In particular, if $T_{\lambda} \neq B_{\lambda}$ and $F_{\lambda}^{j}\left(v_{\lambda}\right)$ lies in $T_{\lambda}$ for some $j \geq 1$, then $J\left(F_{\lambda}\right)$ is a Sierpinski curve.

We remark that case 2 of this theorem does not occur if $n=d=2$ or if $d=1$. In each parameter plane in Figure 1, the exterior red region consists of the parameters for which the Julia set is a Cantor set. We call this region the Cantor set locus. The small red region that contains the origin is the set of parameters for which the Julia set is a Cantor set of circles. We call this region the McMullen domain because McMullen first discovered this type of Julia set [12]. The complement of these two regions is the connectedness locus. The Julia sets for these parameters are connected. The "holes" in the connectedness locus consist of parameter values for which the Julia set is a Sierpiński curve. We call these regions Sierpiński holes.

Note that there are two large Mandelbrot sets along the real axis in the left parameter plane in Figure 1. In the right parameter plane in the same figure, there are three large Mandelbrot sets symmetrically located with respect to the rotation $z \mapsto \nu z$ where $\nu=e^{2 \pi i / 3}$.

With the exception of the $n=d=2$ case, the parameter plane for the family $F_{\lambda}$ contains $n-1$ symmetrically located Mandelbrot sets if $d \neq 1[8]$. We call these sets the principal Mandelbrot sets for the family $F_{\lambda}$. In [8], the existence of these sets was proved for the case $n=d>2$. However, the same proof works if $d \neq 1$ and $n \neq d$. In this paper, we describe the structure of and dynamics on the Julia sets for parameters that lie in the main cardioids of these principal Mandelbrot sets.

If $n=d=2$, there does not exist a principal Mandelbrot set in the parameter plane. In this case, the "tail" of the Mandelbrot set, i.e., the parameter corresponding to $c=-2$ in the Mandelbrot set for $z^{2}+c$, extends to the origin, where the map is just $F_{0}(z)=z^{2}$. So, we do not have a complete Mandelbrot set. Nonetheless, there is a main cardioid in which each parameter has four connecting Fatou components (see the left-hand parameter plane in Figure 5).

If $d=1$, there are no principal Mandelbrot sets in the parameter plane. However, there are $n-1$ distinct cardioid-shaped regions extending from 
the Cantor set locus to the origin (see the right-hand parameter plane in Figure 5). Parameters drawn from these regions have $n+1$ connecting Fatou components.
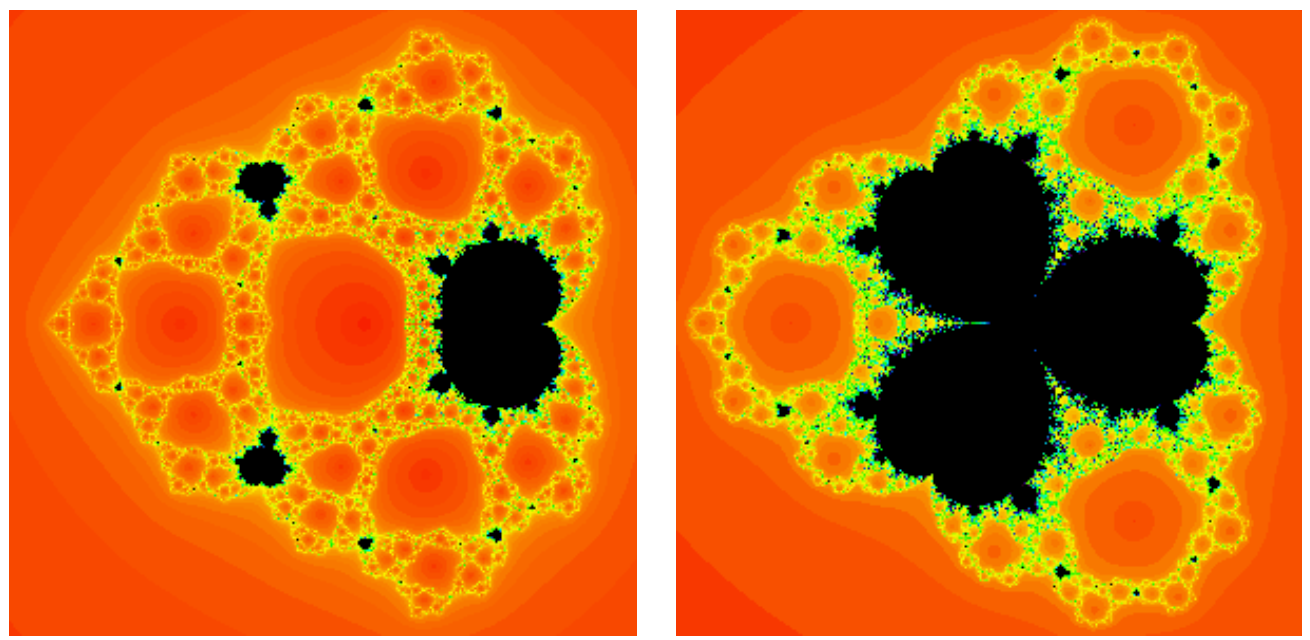

Figure 5: Two parameter planes: $n=d=2$ (left) and $n=4, d=1$ (right).

For the maps that we study, the boundary of the immediate basin of infinity is a simple, closed curve.

Proposition 1. Suppose $\lambda$ lies in the connectedness locus in the parameter plane and $F_{\lambda}$ is hyperbolic on its Julia set. Then the boundary of $B_{\lambda}$ is a simple, closed curve.

Proof: Since $F_{\lambda}$ is hyperbolic on its Julia set, $J\left(F_{\lambda}\right)$ is locally connected (see [13]). In particular, the boundary of $J\left(F_{\lambda}\right)$ is locally connected, so $\partial B_{\lambda}$ is a locally connected set. We need only show that the set $\overline{\mathbb{C}}-\overline{B_{\lambda}}$ is connected.

We argue by contradiction. Suppose that $\overline{\mathbb{C}}-\overline{B_{\lambda}}$ is disconnected. Let $W_{0}$ denote its component that contains the trap door. The second symmetry lemma implies that $W_{0}$ is symmetric under $z \mapsto \omega z$. Also, $\overline{T_{\lambda}}$ is contained in the interior of $W_{0}$ since, if not, there would be a critical point in $\partial T_{\lambda} \cap \partial B_{\lambda}$, which contradicts the assumption that $F_{\lambda}$ is hyperbolic on $J\left(F_{\lambda}\right)$.

At least one other component of $\overline{\mathbb{C}}-\overline{B_{\lambda}}$, say $W_{1}$, is mapped over $W_{0}$. If not, $\partial W_{0}$ would be backward invariant, which cannot happen. Another 
application of the second symmetry implies that $W_{j}=\omega^{j} W_{1}$ is also mapped onto $W_{0}$ for $j=1, \ldots, n+d-1$. We have $n+d$ distinct preimages of $W_{0}$.

However, we claim that there are points in $W_{0}$ that are also mapped into $W_{0}$. To see why, recall that $\partial T_{\lambda}$ is mapped over the entire boundary of $B_{\lambda}$ by $F_{\lambda}$ and that $\partial T_{\lambda}$ lies in $W_{0}$. Thus there is a point $z_{0}$ in $\partial T_{\lambda}$ that is mapped into $\partial W_{0}$. Then there is a neighborhood of $z_{0}$ in $W_{0}$ mapped to a neighborhood of $F_{\lambda}\left(z_{0}\right)$, and hence there are points in this neighborhood that are mapped inside $W_{0}$. We arrive at a contradiction since we have found points in $W_{0}$ that have more than $n+d$ preimages.

\section{Checkerboard Julia Sets}

In this section we present an algorithm for constructing the Julia sets for parameters in the main cardioids of the principal Mandelbrot sets. Theorem 1 follows from this algorithm.

First, consider the case of the principal Mandelbrot set whose main cardioid intersects the positive real axis, and let $[a, b]$ denote the interval of intersection. By considering the graph of $F_{\lambda} \mid \mathbb{R}$ for $\lambda \in(a, b)$, we see that each such map has an attracting fixed point that is real and positive. Hence, for each $\lambda$ from this main cardioid, the map $F_{\lambda}$ also has an attracting fixed point. We denote this fixed point by $p_{\lambda}^{0}$ and its immediate basin of attraction by $C_{\lambda}^{0}$. Since $F_{\lambda}$ is hyperbolic, $\partial C_{\lambda}^{0}$ is a simple, closed curve. Furthermore, if $\lambda \in(a, b)$, the graph of $F_{\lambda} \mid \mathbb{R}$ shows that $C_{\lambda}^{0}$ extends from $\partial B_{\lambda}$ to $\partial T_{\lambda}$. Consequently, $C_{\lambda}^{0}$ extends from $\partial B_{\lambda}$ to $\partial T_{\lambda}$ for all values of $\lambda$ in this main cardioid. The intersection $\partial B_{\lambda} \cap \partial C_{\lambda}^{0}$ consists solely of a repelling fixed point $q_{\lambda}^{0}$ that is real and positive. Similarly, the intersection $\partial T_{\lambda} \cap \partial C_{\lambda}^{0}$ is also just one point that is real and positive. We denote it by $u_{\lambda}^{0}$. Note that $F_{\lambda}\left(u_{\lambda}^{0}\right)=q_{\lambda}^{0}$.

From the second symmetry, we obtain $n+d-1$ other Fatou components that are symmetrically located around the origin. We denote these Fatou components by $C_{\lambda}^{j}$ with $j=1, \ldots, n+d-1$. They are ordered in the coun- 
terclockwise direction. Since each of these components extends from $T_{\lambda}$ to $B_{\lambda}$, we call them connecting (Fatou) components. Some of these Fatou components are immediate basins of attracting cycles, and others are eventually periodic components. The exact configuration of these components is determined by Symmetry Lemma 2 with $\omega=\exp (2 \pi i /(n+d))$. For example, since $F_{\lambda}(\omega z)=\omega^{n} F_{\lambda}(z)$, we have $F_{\lambda}\left(C_{\lambda}^{1}\right)=C_{\lambda}^{n}, F_{\lambda}\left(C_{\lambda}^{2}\right)=C_{\lambda}^{2 n}$, and so forth. In particular, if $n=d=3$, both $C_{\lambda}^{0}$ and $C_{\lambda}^{3}$ are fixed basins, $C_{\lambda}^{1}$ and $C_{\lambda}^{5}$ are mapped to $C_{\lambda}^{3}$, and $C_{\lambda}^{2}$ and $C_{\lambda}^{4}$ are mapped to $C_{\lambda}^{0}$.

Let $p_{\lambda}^{j}=\omega^{j} p_{\lambda}^{0}, q_{\lambda}^{j}=\omega^{j} q_{\lambda}^{0}$, and $u_{\lambda}^{j}=\omega^{j} u_{\lambda}^{0}$. Then both $q_{\lambda}^{j}$ and $u_{\lambda}^{j}$ lie on $\partial C_{\lambda}^{j}$. Also, $p_{\lambda}^{j}$ lies in the interior of $C_{\lambda}^{j}$ and is either periodic or preperiodic (see Figure 6).

For the other $n-2$ main cardioids of the principal Mandelbrot sets, we have a similar structure due to the $(n-1)$-fold symmetry in the parameter plane. More precisely, if $\nu$ is an $(n-1)^{\text {st }}$ root of unity, the orbits of the critical points of $F_{\lambda}$ and $F_{\nu \lambda}$ behave symmetrically with respect to multiplication by some (fractional) power of $\nu$, as was shown immediately following Symmetry Lemma 3. Consequently, the configuration of the basins for $F_{\nu \lambda}$ is similar to that of $F_{\lambda}$.

Recall that $\partial B_{\lambda}$ and $\partial T_{\lambda}$ are simple, closed curves. Since there are no critical points in $\partial B_{\lambda} \cap \partial T_{\lambda}$, these curves do not intersect. Let $A_{\lambda}$ denote the closed annulus bounded by $\partial B_{\lambda}$ and $\partial T_{\lambda}$. Let $I_{\lambda}^{j}$ denote the closed set in $A_{\lambda}$ that is contained in the region located between the open disks $C_{\lambda}^{j}$ and $C_{\lambda}^{j+1}$. Note that the intersection of $I_{\lambda}^{j}$ and $I_{\lambda}^{j+1}$ is the pair of points $q_{\lambda}^{j+1}$ and $u_{\lambda}^{j+1}$. Thus there are four points on the boundary of each $I_{\lambda}^{j}$ that also lie on the boundary of another such set: a pair of points lies in $I_{\lambda}^{j} \cap I_{\lambda}^{j+1}$ and another pair in $I_{\lambda}^{j} \cap I_{\lambda}^{j-1}$. We call the points $q_{\lambda}^{j}$ the outer junction points and the points $u_{\lambda}^{j}$ the inner junction points (see Figure 6).

Proposition 2. $F_{\lambda}$ maps each $I_{\lambda}^{j}$ univalently (except at the junction points) over the region that is the complement of the three sets $B_{\lambda}, F_{\lambda}\left(C_{\lambda}^{j}\right)$, and $F_{\lambda}\left(C_{\lambda}^{j+1}\right)$.

Proof: Since $F_{\lambda}$ is conjugate to $z \mapsto z^{n}$ on $\partial B_{\lambda}$, the portion of $\partial B_{\lambda}$ that 


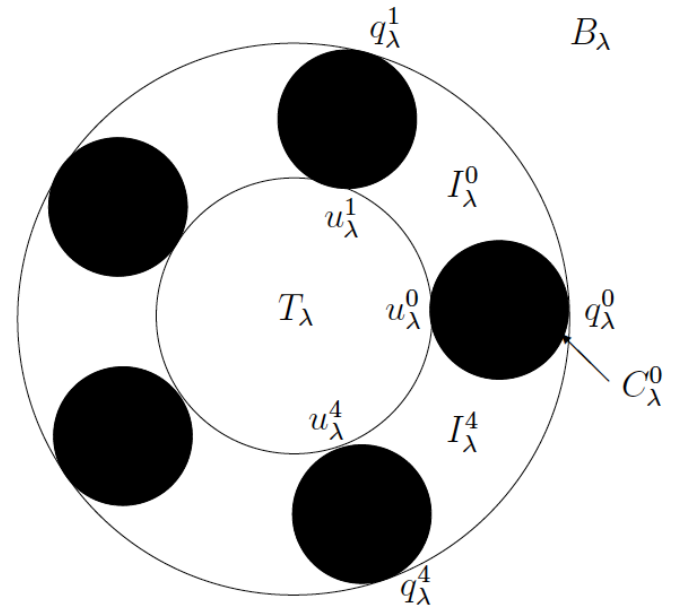

Figure 6: The regions $I_{\lambda}^{j}$ if $n=3$ and $d=2$.

meets $I_{\lambda}^{j}$, i.e., the arc in $\partial B_{\lambda}$ connecting $q_{\lambda}^{j}$ to $q_{\lambda}^{j+1}$, is mapped to an arc in $\partial B_{\lambda}$ that passes through exactly $n+1$ outer junction points. Similarly, the portion of $\partial T_{\lambda}$ that meets $I_{\lambda}^{j}$ is mapped to the complementary arc in $\partial B_{\lambda}$. These two arcs meet at a pair of outer junction points in $\partial B_{\lambda}$. Also, the portion of the boundary of $I_{\lambda}^{j}$ that meets $\partial C_{\lambda}^{j}$ is mapped one-to-one onto the boundary of $F_{\lambda}\left(C_{\lambda}^{j}\right)$ except at the junction points. The junction points are both mapped to the same point. Similarly the other boundary of $I_{\lambda}^{j}$ that lies in $\partial C_{\lambda}^{j+1}$ is mapped onto $\partial F_{\lambda}\left(C_{\lambda}^{j+1}\right)$. Therefore, the boundary of $I_{\lambda}^{j}$ is mapped to the boundary of the three sets $B_{\lambda}, F_{\lambda}\left(C_{\lambda}^{j}\right)$, and $F_{\lambda}\left(C_{\lambda}^{j+1}\right)$. Since there are no critical points in $I_{\lambda}^{j}$, the result follows.

We call the two arcs in $I_{\lambda}^{j}$ that lie in the boundaries of $C_{\lambda}^{j}$ and $C_{\lambda}^{j+1}$ the internal boundary components of $I_{\lambda}^{j}$. By Proposition 2, there must be a preimage of $T_{\lambda}$ in each $I_{\lambda}^{j}$. Moreover, the boundary of this preimage must meet each internal boundary component of $I_{\lambda}^{j}$ in exactly one point, namely the preimage of the inner junction points lying in the portions of the boundary of $F_{\lambda}\left(C_{\lambda}^{j}\right)$ and $F_{\lambda}\left(C_{\lambda}^{j+1}\right)$ that lie in $I_{\lambda}^{j}$. Thus the preimage of $T_{\lambda}$ in each $I_{\lambda}^{j}$ is an open region whose boundary meets exactly one point in each of the boundaries of the connecting Fatou components that are adjacent to $I_{\lambda}^{j}$ (see 
Figure 7).

The preimage of $T_{\lambda}$ separates $I_{\lambda}^{j}$ into two pieces: an external piece that abuts $\partial B_{\lambda}$ and an internal piece that abuts $\partial T_{\lambda}$. The external piece is mapped by $F_{\lambda}$ over the portion of $A_{\lambda}$ that stretches from $F_{\lambda}\left(C_{\lambda}^{j}\right)$ to $F_{\lambda}\left(C_{\lambda}^{j+1}\right)$ in the counterclockwise direction. Since $F_{\lambda}(\omega z)=\omega^{n} F_{\lambda}(z)$, this region is mapped over exactly $n$ of the $I_{\lambda}^{i}$ and $n-1$ of the $C_{\lambda}^{i}$. Similarly, the internal piece is mapped over exactly $d$ of the $I_{\lambda}^{i}$ and $d-1$ of the $C_{\lambda}^{j}$. So each of $I_{\lambda}^{j}$ can be further subdivided as shown in Figure 7. The portion of $I_{\lambda}^{j}$ lying outside the preimage of $T_{\lambda}$ has $n-1$ preimages of the connecting components, and the internal portion has $d-1$ such preimages. Between each preimage including $C_{\lambda}^{j}$ and $C_{\lambda}^{j+1}$, there is a region that is mapped univalently onto one of the $I_{\lambda}^{k}$ 's. Hence there is a preimage of each of the sets just constructed in each of these smaller regions (see Figure 7).
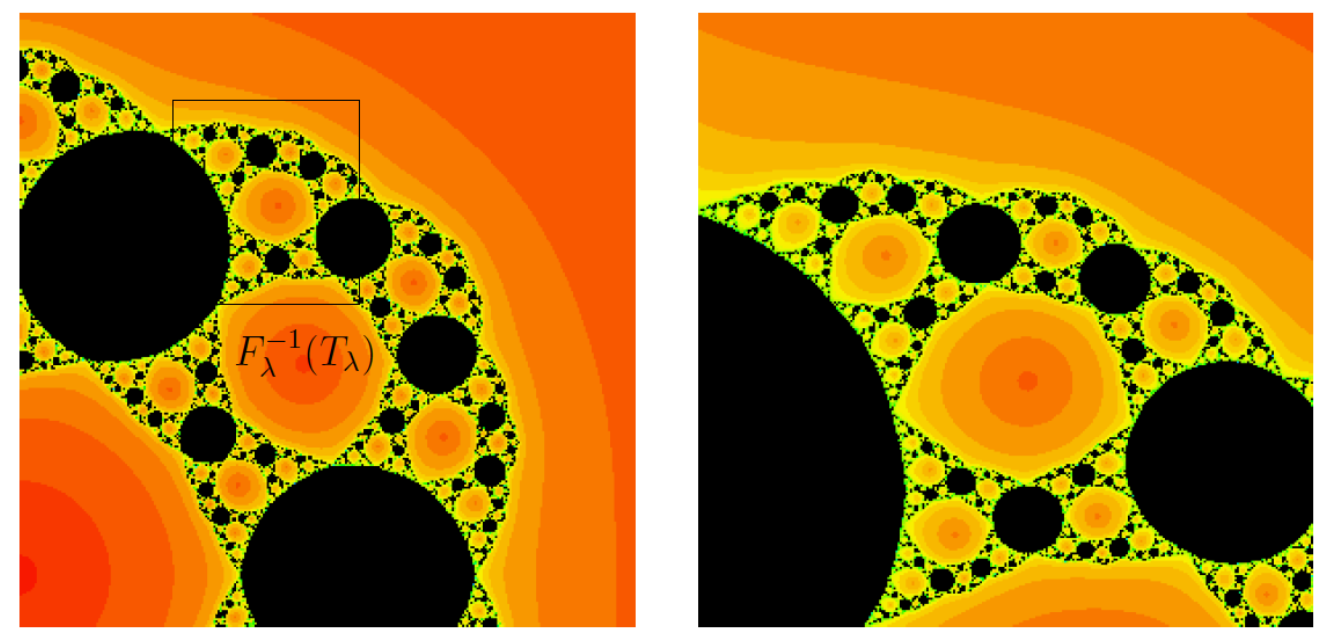

Figure 7: The regions $I_{\lambda}^{j}$ if $n=3$ and $d=2$.

Continuing in this fashion, we always find the same picture in each region bounded by $k^{\text {th }}$ and earlier preimages of $T_{\lambda}$ and $k^{\text {th }}$ and earlier preimages of the $C_{\lambda}^{j}$ 's. It is a central $(k+1)^{\text {st }}$ preimage of $T_{\lambda}$ flanked by $n-1(k+1)^{\text {st }}$ preimages of the connecting components on one side and $d-1$ other $(k+1)^{\text {st }}$ preimages on the other side. 
However, this construction does not give the entire Julia set of $F_{\lambda}$. Indeed, the portion of the Julia set produced thus far contains only preimages of the boundaries of $B_{\lambda}$ and the $C_{\lambda}^{j}$. None of these preimages contain any periodic points; the only periodic points here lie in $\partial B_{\lambda}$ and $\partial C_{\lambda}^{j}$. So there must be more to the Julia set.

To complete the construction of the Julia set, note that each closed region $I_{\lambda}^{j}$ is almost mapped univalently over the union of all of the $I_{\lambda}^{k} \mathrm{~s}$. The map is univalent except at the four junction points. One pair of junction points is mapped to an outer junction point in the image, and the other pair is mapped to a different outer junction point. We can use symbolic dynamics to identify each point in the Julia set. Let $\Sigma$ denote the set of sequences $\left(s_{0}, s_{1}, s_{2}, \ldots\right)$ where each $s_{j}$ is one of the integers $0,1, \ldots, n+d-1$. We identify each point in $J\left(F_{\lambda}\right)$ with a point in $\Sigma$ by assigning to each $z \in J\left(F_{\lambda}\right)$ its itinerary $S(z)=\left(s_{0}, s_{1}, s_{2}, \ldots\right)$ where $s_{k}=j$ if $F_{\lambda}^{k}(z) \in I_{\lambda}^{j}$. However, infinitely many points are assigned to a pair of sequences. The points $q_{\lambda}^{j}$ and $u_{\lambda}^{j}$ each have a pair of sequences attached to them since these points reside in two of the $I_{\lambda}^{j}$ ss. For example, $S\left(q_{\lambda}^{0}\right)=(\overline{0})=(\overline{n+d-1})$ and $S\left(u_{\lambda}^{0}\right)=(0, \overline{n+d-1})=(n+d-1, \overline{0})$. Similarly, any point that is eventually mapped onto a $q_{\lambda}^{j}$ or a $u_{\lambda}^{j}$ also has a pair of itineraries, e.g., the itineraries $\left(s_{0}, \ldots, s_{k}, 0, \overline{n+d-1}\right)$ and $\left(s_{0}, \ldots, s_{k}, n+d-1, \overline{0}\right)$ correspond to the same points.

We let $\Sigma^{\prime}$ denote the sequence space with the above identifications and endow $\Sigma^{\prime}$ with the quotient topology. Since each $I_{\lambda}^{j}$ is mapped univalently (except at the junction points) over the union of the $I_{\lambda}^{k}$ and the Julia set is contained in this union, standard arguments then show that the Julia set is homeomorphic to $\Sigma^{\prime}$. The subsets $\Sigma_{j}^{\prime}$ of $\Sigma^{\prime}$ consisting of all sequences that start with the digit $j$ correspond to points in $I_{\lambda}^{j} \cap J\left(F_{\lambda}\right)$, and they are homeomorphic to $\Sigma_{k}^{\prime}$. It is important to note that the dynamics on these sets are not the same even though they are homeomorphic. We have described the topological structure of each $I_{\lambda}^{j} \cap J\left(F_{\lambda}\right)$, and this description implies Theorem 1 (see [2] for a similar argument). 


\section{Dynamical Invariants}

In this section we prove Theorems 2 and 3. Let $\nu=\exp (2 \pi i /(n-1))$. We show that two maps drawn from the main cardioids of different principal Mandelbrot sets are conjugate on their Julia sets if and only if the cardioids are located symmetrically under either the maps $z \mapsto \nu^{j(d+1)} z$ or $z \mapsto \nu^{j(d+1)} \bar{z}$ for some integer $j$.

We first observe that it suffices to prove this result for the special maps whose parameter is the center of these main cardioids. The set of critical points is invariant under the map, so the critical points are either periodic or preperiodic. The following proposition follows from the work of Mané, Sad, and Sullivan [10].

Proposition 3. Suppose $\lambda$ lies at the center of the main cardioid of one of the principal Mandelbrot sets and that $\mu$ lies in the same cardioid. Then $F_{\lambda}$ and $F_{\mu}$ are quasiconformally conjugate on their Julia sets.

Remark. It is not true that $F_{\lambda}$ and $F_{\mu}$ are globally conjugate since $F_{\lambda}$ has a superattracting cycle while the attracting cycle for $F_{\mu}$ need not be superattracting.

By Proposition 3, we need only consider parameters that lie at the centers of the main cardioids of the principal Mandelbrot sets. So for the remainder of this section, we assume that $\lambda$ and $\mu$ are centers. Then $\mu=\nu^{j} \lambda$ for some $j \in \mathbb{Z}$. The proof of one direction of Theorem 2 is straightforward:

Proposition 4. If $\mu=\nu^{j(d+1)} \lambda$ or $\mu=\nu^{j(d+1)} \bar{\lambda}$ for some integer $j$, then $F_{\mu}$ is conjugate to $F_{\lambda}$.

Proof: Let $\mu=\nu^{j(d+1)} \lambda$, then

$$
F_{\mu}\left(\nu^{j} z\right)=\nu^{j n} z^{n}+\frac{\lambda \nu^{j(d+1)}}{\nu^{j d} z^{d}}=\nu^{j}\left(z^{n}+\frac{\lambda}{z^{d}}\right)=\nu^{j} F_{\lambda}(z) .
$$

So $F_{\lambda}$ is conjugate to $F_{\mu}$ via the linear map $z \mapsto \nu^{j} z$.

By Symmetry Lemma $1, F_{\lambda}$ and $F_{\bar{\lambda}}$ have conjugate dynamics. So if $\mu=\nu^{j(d+1)} \bar{\lambda}$, then $F_{\mu}$ is conjugate to $F_{\bar{\lambda}}$ and hence also to $F_{\lambda}$. 
From Proposition 4, we know that all centers whose parameters are of the form $\nu^{k} \lambda$ or $\nu^{k} \bar{\lambda}$ where $k=j(d+1) \bmod (n-1)$ have conjugate dynamics. That is, any two main cardioids that are located symmetrically with respect to either rotation by $\nu^{d+1}$ or complex conjugation have conjugate dynamics. Note that $\nu^{d+1}=\nu^{n-1} \nu^{d+1}=\nu^{n+d}$, so we can say that any two cardioids that are located symmetrically with respect to either rotation by $\nu^{n+d}$ or complex conjugation have conjugate dynamics.

Using basic facts about the greatest common divisor of two numbers, we can restate this relationship among the centers with conjugate dynamics in terms of the greatest common divisor $g$ of $d+1$ and $n-1$. In fact, all centers whose parameters are of the form $\nu^{k} \lambda$ or $\nu^{k} \bar{\lambda}$ where $k$ is an integer multiple of $g$ have conjugate dynamics.

Now we show that these symmetrically located centers are the only centers with conjugate dynamics. First we define the minimum rotation number for parameters in the main cardioids of the principal Mandelbrot sets. For each such parameter we have $n+d$ connecting components $C_{\lambda}^{j}$ with $j$ defined mod $n+d$, and the $C_{\lambda}^{j}$ are ordered in the counterclockwise direction as $j$ increases. Each of these connecting components is mapped two-to-one onto another such component since each contains a unique critical point (see Figure 6).

Suppose $F_{\lambda}\left(C_{\lambda}^{j}\right)=C_{\lambda}^{k}$. We define the rotation number $\rho_{j}$ of $C_{\lambda}^{j}$ to be the value of $k-j$ that is closest to 0 for any $k \bmod n+d$. Note that it is possible for $k$ to be negative (see Figure 8). For example, if $F_{\lambda}\left(C_{\lambda}^{0}\right)=C_{\lambda}^{n+d-1}$, then the rotation number of $C_{\lambda}^{0}$ would be -1 since $C_{\lambda}^{n+d-1}=C_{\lambda}^{-1}$. We say that $C_{\lambda}^{j}$ is rotated through $k-j$ components if $\rho_{j}=k$. We then define the minimum rotation number $\rho(\lambda)$ for $F_{\lambda}$ to be the minimum value of $\left|\rho_{j}\right|$ over all $j$. For example, if $F_{\lambda}$ has an attracting fixed point in some $C_{\lambda}^{j}, \rho(\lambda)=0$. If there is no such attracting fixed point, then $\rho(\lambda)>0$.

Proposition 5. Let $\lambda$ and $\mu$ be centers of main cardioids of principal Mandelbrot sets. Then $F_{\lambda}$ is conjugate to $F_{\mu}$ if and only if $\rho(\lambda)=\rho(\mu)$. Equivalently, $\mu=\nu^{k} \lambda$ or $\mu=\nu^{k} \bar{\lambda}$ where $k=j(d+1) \bmod (n-1)$ for some integer $j$. The conjugating map is either a rotation about the origin or a rotation 


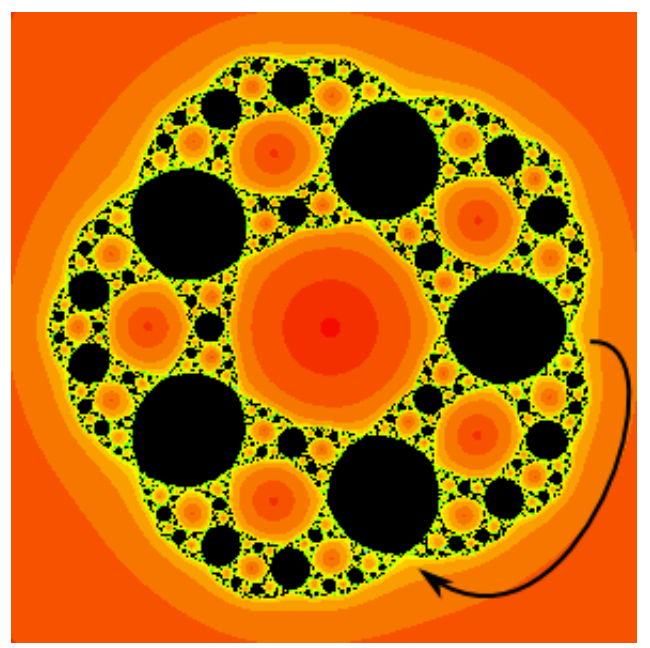

Figure 8: A $C_{\lambda}^{j}$ with $\rho_{j}=-1$.

\section{followed by complex conjugation.}

Proof: First suppose that two such centers $\lambda$ and $\mu$ have different minimum rotation numbers. Then $F_{\lambda}$ and $F_{\mu}$ cannot be conjugate on their Julia sets. To see why, recall that the connecting components each touch $\partial B_{\lambda}$ at exactly one point. Now $\partial B_{\lambda}$ must be sent to itself by any conjugacy between $F_{\lambda}$ and $F_{\mu}$ since this set is the only invariant subset of the Julia set that touches the boundaries of all of the connecting components. Thus the ordering of the $C_{\lambda}^{j}$ is either preserved or reversed by the conjugacy, i.e., either the conjugacy rotates the connecting components in one direction or the other, or the conjugacy first applies complex conjugation followed by some rotation. In either case, if the minimum rotation numbers of $F_{\lambda}$ and $F_{\mu}$ are different, then such a conjugacy cannot exist.

To prove the converse, we consider the set of critical points, $c_{\lambda}^{0}, \ldots, c_{\lambda}^{n+d-1}$, of $F_{\lambda}$ where $\lambda$ is the center of a main cardioid of a principal Mandelbrot set. The point $c_{\lambda}^{j}$ is the unique critical point that lies in the connecting component $C_{\lambda}^{j}$. We note that this set of critical points is invariant under $F_{\lambda}$.

Suppose $F_{\lambda}$ and $F_{\mu}$ have the same minimum rotation number $m$. By assumption, there is at least one critical point $c_{\lambda}^{j}$ for $F_{\lambda}$ for which either 
$F_{\lambda}\left(c_{\lambda}^{j}\right)=c_{\lambda}^{j+m}=\omega^{m} c_{\lambda}^{j}$ or $F_{\lambda}\left(c_{\lambda}^{j}\right)=c_{\lambda}^{j-m}=\omega^{-m} c_{\lambda}^{j} . \quad\left(\right.$ Recall that $\left.\omega^{n+d}=1.\right)$ There is also a critical point $c_{\mu}^{i}$ for $F_{\mu}$ for which either $F_{\mu}\left(c_{\mu}^{i}\right)=c_{\mu}^{i+m}=\omega^{m} c_{\mu}^{i}$ or $F_{\mu}\left(c_{\mu}^{i}\right)=c_{\mu}^{i-m}=\omega^{-m} c_{\mu}^{i}$.

We consider the first case for $\lambda$ and $\mu$, i.e., where the rotation numbers $m$ for both critical points are positive. Since $\mu=\nu^{k} \lambda$ for some $k, F_{\mu}$ is conjugate to the map $z \mapsto \omega^{k} F_{\lambda}(z)$ by the rotation $z \mapsto \eta^{k} z$ where

$$
\eta=\exp (2 \pi i /((n+d)(n-1)))
$$

(see the paragraphs that follow Symmetry Lemma 3). So there must be a critical point for $\omega^{k} F_{\lambda}$ that corresponds to $c_{\mu}^{i}$ and that is also rotated by $\omega^{m}$ when $\omega^{k} F_{\lambda}$ is applied to it. But any critical point of $\omega^{k} F_{\lambda}$ must also be a critical point for $F_{\lambda}$. Suppose that $\omega^{\ell} c_{\lambda}^{j}$ is the critical point for $\omega^{k} F_{\lambda}$ that corresponds to $c_{\mu}^{i}$. Then we have

$$
\omega^{k} F_{\lambda}\left(\omega^{\ell} c_{\lambda}^{j}\right)=\omega^{m} \omega^{\ell} c_{\lambda}^{j}=\omega^{\ell} \omega^{m} c_{\lambda}^{j}=\omega^{\ell} F_{\lambda}\left(c_{\lambda}^{j}\right) .
$$

But

$$
\omega^{k} F_{\lambda}\left(\omega^{\ell} c_{\lambda}^{j}\right)=\omega^{k+\ell n} F_{\lambda}\left(c_{\lambda}^{j}\right) .
$$

Therefore we have $\ell=k+\ell n \bmod (n+d)$.

Consequently, $\omega^{\ell} F_{\lambda}(z)=\omega^{k+\ell n} F_{\lambda}(z)$, and using Symmetry Lemma 2, we obtain

$$
\omega^{\ell} F_{\lambda}(z)=\omega^{k} F_{\lambda}\left(\omega^{\ell} z\right)
$$

for all $z \in \mathbb{C}$. So $F_{\lambda}$ is conjugate to $\omega^{k} F_{\lambda}$ via the map $z \mapsto \omega^{\ell} z$. Therefore $F_{\lambda}$ is also conjugate to $F_{\mu}$ via a linear map of the form $z \mapsto \eta \omega^{\ell} z$ and $\mu=\nu^{k} \lambda$ where $k=j(d+1) \bmod (n-1)$.

The proof for the case where both rotation numbers $m$ are negative is exactly the same.

To prove the second case, the case where the rotations go in opposite directions, we simply conjugate $F_{\mu}$ to $F_{\bar{\mu}}$ by complex conjugation and then invoke the first case.

As a consequence of Propositions 3 and 5, this result extends to all parameters in the main cardioids of any of the principal Mandelbrot sets. 
Corollary. Let $\lambda$ and $\mu$ be any parameters drawn from the main cardioids of any two prinicpal Mandelbrot sets. Then $F_{\lambda}$ and $F_{\mu}$ are conjugate on their Julia sets if and only if $\rho(\lambda)=\rho(\mu)$.

Now we can determine exactly which main cardioids of the principal Mandelbrot sets have conjugate dynamics and the precise number of different conjugacy classes. Let $\mathcal{M}_{0}$ denote the main cardioid of the principal Mandelbrot set that intersects the positive real axis, and denote the remaining main cardioids of principal Mandelbrot sets by $\mathcal{M}_{j}$ where the ordering is in the counterclockwise direction. We write $\mathcal{M}_{j} \equiv \mathcal{M}_{k}$ if the parameters at the centers of $\mathcal{M}_{j}$ and $\mathcal{M}_{k}$ have conjugate dynamics. Let $g$ be the greatest common divisor of $n-1$ and $d+1$. As we proved in this section, the principal Mandelbrot sets with dynamics conjugate to the dynamics of $\mathcal{M}_{k}$ are those obtained by successive rotations in the parameter plane by $z \mapsto \nu^{j g} z$ or by these rotations followed by complex conjugation. In particular, we have $\mathcal{M}_{0} \equiv \mathcal{M}_{j g}$ for all integers $j$.

Theorem. If the greatest common divisor $g$ is even, there are $1+g / 2$ different conjugacy classes among the $\mathcal{M}_{j}$. If $g$ is odd, there are $(g+1) / 2$ distinct such conjugacy classes.

Proof: First suppose that $g=1$. Then all maps drawn from the $\mathcal{M}_{j}$ have conjugate dynamics, so we have $1=(g+1) / 2$ conjugacy classes.

Now suppose $g>1$. We claim that $\mathcal{M}_{k} \not \equiv \mathcal{M}_{0}$ for any $k$ with $0<k<g$. If not, then maps at the centers of $\mathcal{M}_{0}$ and some $\mathcal{M}_{k}$ would be conjugate by $z \mapsto \bar{z}$ followed possibly by a rotation. But then $\mathcal{M}_{k} \equiv \mathcal{M}_{-k}$ via $z \mapsto \bar{z}$. Also, $\mathcal{M}_{g} \equiv \mathcal{M}_{-g}$ by $z \mapsto \bar{z}$. Therefore we have $\mathcal{M}_{-g} \equiv \mathcal{M}_{-k}$ by a rotation, which would imply that the greatest common divisor is smaller than $g$. So none of the centers of the $\mathcal{M}_{k}$ with $0<k<g$ have dynamics conjugate to the center of $\mathcal{M}_{0}$.

If $g$ is even, we consider $\mathcal{M}_{k}$ where $0<k<g / 2$. We have $\mathcal{M}_{k} \equiv$ $\mathcal{M}_{-k}$ by complex conjugation. Moreover $\mathcal{M}_{-k} \equiv \mathcal{M}_{g-k}$ since these sets are symmetric under the rotation $z \mapsto \nu^{g} z$, so $\mathcal{M}_{k} \equiv \mathcal{M}_{g-k}$. On the other hand, we cannot have $\mathcal{M}_{k} \equiv \mathcal{M}_{j}$ for any other $j$ with $0<j<g$ via rotation 
by $z \mapsto \nu^{g} z$ or by complex conjugation coupled with a rotation, so the principal Mandelbrot sets with dynamics conjugate to those in $\mathcal{M}_{k}$ are just the rotations of $\mathcal{M}_{k}$ together with their complex conjugates. The number of such conjugacy classes is $g / 2-1$. We have $\mathcal{M}_{g / 2} \equiv \mathcal{M}_{-g / 2}$ by the rotation $z \mapsto \nu^{-g} z$ as well as by complex conjugation. So $\mathcal{M}_{g / 2}$ lies in a conjugacy class that is distinct from the classes of the $\mathcal{M}_{k}$ with $0 \leq k \leq g / 2$. The conjugacy class of $\mathcal{M}_{0}$ has not yet been counted. Combining all of these classes, we obtain a total of $1+g / 2$ distinct conjugacy classes.

If $g$ is odd, we count in exactly the same way except that we do not have a conjugacy class that corresponds to $\mathcal{M}_{g / 2}$ in this case.

See Figure 9 for the three orbit diagrams that arise if $n=13$ and $d=7$. In Figure 10, we consider the case where $n=11$ and $d=4$, and group the Mandelbrot sets whose centers have conjugate dynamics.
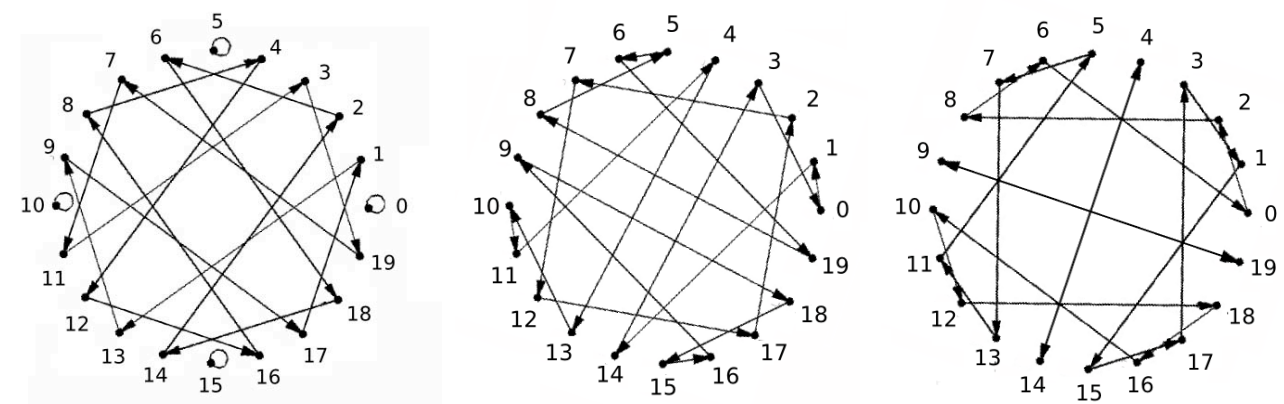

Figure 9: If $n=13$ and $d=7$, then $g=4$, and consequently, there are three conjugacy classes. This figure contains one orbit diagram for each of the three classes.

\section{A Group Action}

Since the conjugacies among the $\mathcal{M}_{k}$ arise from reflective and rotational symmetries, we can count the number of conjugacy classes by viewing them 


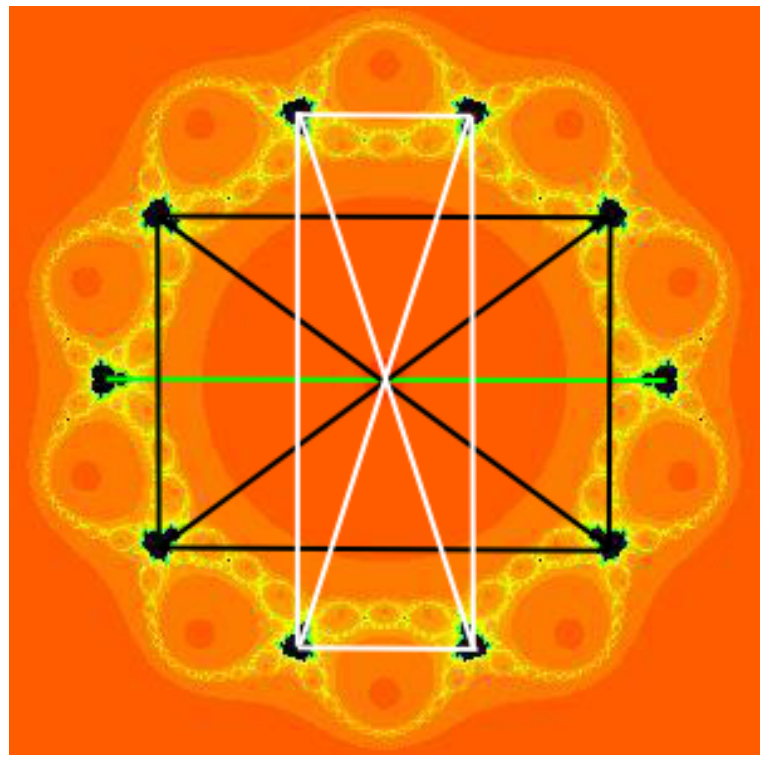

Figure 10: If $n=11$ and $d=4$, then $g=5$, and consequently, there are three conjugacy classes. The parameters with conjugate dynamics are connected by segments of the same color, e.g., the four Mandelbrot sets connected by white segments all have conjugate dynamics.

as orbits of the action of a dihedral group on the set $\left\{\mathcal{M}_{k}\right\}$, viewed as the vertices of a regular $(n-1)$-gon.

Let $a=(n-1) / g$. We claim that the natural group that produces these orbits is $D_{2 a}$, the group of symmetries of a regular $a$-gon. Let $s$ be the generator of $D_{2 a}$ corresponding to reflection and $r$ be the generator corresponding to rotation. We define the action of $D_{2 a}$ on $\left\{\mathcal{M}_{k}\right\}$ by

$$
\begin{aligned}
s \mathcal{M}_{k} & =\mathcal{M}_{-k \bmod n-1} \\
r \mathcal{M}_{k} & =\mathcal{M}_{k+g \bmod n-1}
\end{aligned}
$$

These rules produce a well-defined $D_{2 a}$ action, and since the actions on $\left\{\mathcal{M}_{k}\right\}$ by $s$ and $r$ are exactly complex conjugation and rotation by $z \mapsto \nu^{g} z$, respectively, the orbits of this action correspond exactly to the conjugacy classes among the $\mathcal{M}_{k}$. 
By Burnside's Lemma, the number of orbits is

$$
\frac{1}{\left|D_{2 a}\right|} \sum_{x \in D_{2 a}}|\operatorname{fix}(x)|
$$

where fix $(x)=\left\{\mathcal{M}_{i} \in\left\{\mathcal{M}_{k}\right\}: x \mathcal{M}_{i}=\mathcal{M}_{i}\right\}$.

The group $D_{2 a}$ has $2 a$ elements, and each can be written as $r^{j}$ or $s r^{j}$ with $0 \leq j<a$. The identity fixes all $n-1$ elements of $\left\{\mathcal{M}_{k}\right\}$, and $r^{j}$ fixes none for $0<j<a$. Thus the number of orbits is

$$
\frac{1}{2 a}\left(n-1+\sum_{j=0}^{a-1}\left|\operatorname{fix}\left(s r^{j}\right)\right|\right)
$$

An element of the form $s r^{j}$ rotates each $\mathcal{M}_{k}$ by $z \mapsto \nu^{j g} z$ and then reflects it about the real axis. Equivalently, it reflects the $\mathcal{M}_{k}$ through some axis of symmetry of the set viewed as a regular $(n-1)$-gon. Thus, if $n-1$ is odd, every such axis passes through exactly one of the $\mathcal{M}_{k}$, and thus $\left|\operatorname{fix}\left(s r^{j}\right)\right|=1$ for all $j$. The formula above then shows the number of conjugacy classes is $(n-1+a) / 2 a=(g+1) / 2$.

If $n-1$ is even, half of the axes of symmetry pass through two of the $\mathcal{M}_{k}$, and half pass through none. Thus $s r^{j}$ fixes either two or zero of the $\mathcal{M}_{k}$. There exists a $j$ such that $s r^{j}$ fixes none of the $\mathcal{M}_{k}$ if and only if there is some $i$ such that $s r^{j} \mathcal{M}_{i}=\mathcal{M}_{i+1 \bmod n-1}$, i.e., the axis of reflection passes between $\mathcal{M}_{i}$ and $\mathcal{M}_{i+1 \bmod n-1}$ for some $i$. For such an $i, r^{j} \mathcal{M}_{i}=s^{-1} \mathcal{M}_{i+1 \bmod n-1}=$ $\mathcal{M}_{-i-1 \bmod n-1}$ which equals $\mathcal{M}_{i+j g \bmod n-1}$ by the definition of the action of $r$. Thus $-i-1 \equiv i+j g \bmod n-1$, and hence, $2 i+j g+1 \equiv 0 \bmod n-1$. If either $j$ or $g$ is even, this equality is impossible since $n-1$ is even, and therefore, $s r^{j}$ must fix two of the $\mathcal{M}_{k}$. If $j$ and $g$ are both odd, however, any $i$ with $i \equiv(-j g-1) / 2 \bmod n-1$ satisfies the congruence, and thus $s r^{j}$ fixes none of the $\mathcal{M}_{k}$.

Therefore, if $n-1$ and $g$ are even, then $\left|\operatorname{fix}\left(s r^{j}\right)\right|=2$ for all $j$, and the number of conjugacy classes is $(n-1+2 a) / 2 a=1+g / 2$. If $n-1$ is even and $g$ is odd, $\left|\operatorname{fix}\left(s r^{j}\right)\right|$ equals 2 if $j$ is even, and 0 if $j$ is odd. Hence, there are $(n-1+a) / 2 a=(g+1) / 2$ conjugacy classes. 
Finally, if $n-1$ is odd, $g$ must be odd, so the possible cases really depend only on the parity of $g$ and not of $n-1$. Hence, the number of conjugacy classes is $(g+1) / 2$ if $g$ is odd and $1+g / 2$ if $g$ is even.

\section{References}

[1] Blanchard, P., Devaney, R. L., Look, D. M., Seal, P., and Shapiro, Y. Sierpiński Curve Julia Sets and Singular Perturbations of Complex Polynomials. Ergodic Theory and Dynamical Systems, 25 (2005), 1047-1055.

[2] Çilingir, F., Devaney, R. L., and Russell, E. D. Extending external rays throughout the Julia sets of rational maps. Journal of Fixed Point Theory and Applications, appeared online March 10, 2011.

[3] Devaney, R. L. and Look, D. M. Buried Sierpiński Curve Julia Sets. Discrete and Continuous Dynamical Systems 13 (2005), 1035-1046.

[4] Devaney, R. L., Look, D. M. A Criterion for Sierpiński Curve Julia Sets. Topology Proceedings 30 (2006), 163-179.

[5] Devaney, R. L., Look, D. M., and Uminsky, D. The Escape Trichotomy for Singularly Perturbed Rational Maps. Indiana University Mathematics Journal 54 (2005), 1621-1634.

[6] Devaney, R. L. and Pilgrim, K. Dynamic Classification of Escape Time Sierpinski Curve Julia Sets. Fundamenta Mathematicae 202 (2009), 181-198.

[7] Devaney, R. L. Cantor Necklaces and Structurally Unstable Sierpiński Curve Julia Sets for Rational Maps. Qualitative Theory of Dynamical Systems 5 (2004), 337-359. 
[8] Devaney, R. L. Baby Mandelbrot Sets Adorned with Halos in Families of Rational Maps. In Complex Dynamics: Twenty-Five Years after the Appearance of the Mandelbrot Set. American Math Society, Contemporary Math 396 (2006), 37-50.

[9] Devaney, R. L. A Myriad of Sierpiński Curve Julia Sets. In Difference Equations, Special Functions, and Orthogonal Polynomials. World Scientific, 2007, 131-148.

[10] Mañé, R., Sad, P., and Sullivan, D., On the dynamics of rational maps. Annales Scientifiques de l'École Normale Supérieure. Quatrième Série 16, 1983, 193-217.

[11] McMullen, C. Automorphisms of Rational Maps. Holomorphic Functions and Moduli. Vol. 1. Math. Sci. Res. Inst. Publ. 10. Springer, New York, 1988.

[12] McMullen, C. The Classification of Conformal Dynamical Systems. Current Developments in Mathematics. International Press, Cambridge, MA, (1995) 323-360.

[13] Milnor, J. Dynamics in One Complex Variable, 3ed, Princeton University Press, 2006.

[14] Milnor, J. and Tan Lei. A "Sierpiński Carpet" as Julia Set. Appendix F in Geometry and Dynamics of Quadratic Rational Maps. Experiment. Math. 2 (1993), 37-83.

[15] Whyburn, G. T. Topological characterization of the Sierpiński curve, Fund. Math. 45 (1958), 320-324. 